\title{
Resecciones y Suturas en el Trauma Intestinal Contuso
}

\author{
O Paredes, L Madelaire, J Est Domínguez
}

\section{RESUMEN}

En un estudio observacional retrospectivo de corte transverso de un grupo de pacientes con diagnóstico de lesión intestinal en traumatismo abdominal cerrado; operados en el Servicio de Emergencias Médicas de Asunción-Paraguay se concluyó que:

1. La contusión abdominal puede requerir procedimientos de sutura y-o $C$ ya por su apertura a cavidad abdominal libre o por comprometimiento de los vasos mesentéricos.

2. En contusiones abdominales asociadas a lesión intestinal es más probable la lesión del intestino delgado que del colon.

3. La mortalidad de la serie está mayormente asociada a complicaciones de lesiones no intestinales.

Palabras clave: Lesiones intestinales, Trauma abdominal contuso.

\section{ABSTRACT}

In a retrospective observational transverse study of a group of patients with bowel injury in blunt abdominal trauma, operated in the Emergency Medical Service of Asuncion-Paraguay we concluded that:

1. Abdominal contusion may require procedures involving bowel resection and suture, for its free abdominal cavity or the mesenteric vessels compromise.

2. In abdominal contusions associated with intestinal injury it is more likely for the small intestine to be injured than the colon.

3. The mortality of the series is mostly associated with complications of injuries not due to intestinal wounds.

Keywords: Bowel injury, Blunt abdominal trauma.

How to cite this article: Paredes $O$, Madelaire L, Domínguez JE. Resecciones y Suturas en el Trauma Intestinal Contuso. Panam J Trauma Critical Care Emerg Surg 2012;1(2):68-71.

\section{Source of support $\mathrm{Nil}$}

Conflict of interest None

\section{INTRODUCCIÓN}

A unque lo habitual es pensar en lesiones intestinal es abiertas, a consecuencia de heridas penetrantes, el traumatismo contuso también puede ser su causa. ${ }^{1} \mathrm{~L}$ a simple presentación en ese contexto ya sugiere la violencia con que el agente tuvo que haber actuado o la intensidad del mecanismo de trauma. ${ }^{1,2}$

L a presentación de lesión intestinal abierta en el contexto del paciente contuso es rara y en general su diagnóstico no es evocado, ya que aunque la sagacidad clínica pueda sugerirla, ella es más bien descubierta en las laparotomías de pacientes que son explorados con otras indicaciones. ${ }^{1,3}$

La literatura en general no sugiere un Gold estándar para su diagnostico pero en general afirman que un esquema de manejo protocolizado en cuanto a observación clínica y métodos de diagnostico bien llevados hacen que los mismo puedan ser tratados en tiempo oportuno. ${ }^{1,4}$

A unque se citan lesiones intestinales en casos de traumatismos contusos aparentemente mínimos, ${ }^{1}$ la presentación clásica suele ser la de un paciente con antecedente de trauma cerrado importante donde se descubre líquido libre en el abdomen. L a sugerencia primera es considerar ese líquido como sangre y al permanecer hemodinámicamente estable, no se sugiere la laparotomía de entrada. A partir de esta primera impresión se solicitan varios estudios variables en su rendimiento en cuanto a su sensibilidad y especificidad pero ninguna con un rendimiento del $100 \%$, teniendo entonces la probabilidad más cercana a este diagnostico, la implementación del lavado peritoneal diagnóstico; si se considera una relación costo/beneficio. ${ }^{1}$

La presentación también puede ser objetivada a partir de un cuadro séptico de origen abdominal que por unidad clínica, propicia la exploración abdominal.

Se quiere destacar entonces que el control seriado del abdomen del paciente consiente y colaborador es un elemento importante para su diagnóstico, y para los casos de cuadros no aclarados con liquido libre, el LPD sería el elemento que pudiera despejarnos de dudas. ${ }^{1}$

\section{MATERIAL Y METODOS}

Sobre un total de 58 paciente tratados en el servicio de Emergencias M édicas deA sunción-Paraguay diagnosticados como con lesiones intestinales en trauma cerrado de abdomen en el transcurso de 4 años 2007-2010); se estableció un análisis de las historias clínicas a fin de determinar su relación y 10 asociación según datos demográficos, mecanismo de trauma, lesión discriminada según intestino afectado y mortalidad.

\section{OBJETIVOS}

1. Determinar las etiologías relacionadas a lesiones intestinales por trauma contuso de abdomen.

2. Determinar la prevalencia de lesiones intestinales en forma discriminada (delgado y grueso).

3. Describir características relacionadas a su manejo y evolución.

\section{RESULTADOS}

Según edad y sexo: del total de 58 pacientes con diagnóstico de lesión intestinal, 18 no ameritaron procedimiento

Paper presented in Free Research Competition at the 24th Annual Congress of SPT in Asuncion, Paraguay on November 2011. 
específico por considerarse que la injuria abarcaba solo planos superficiales.

Existieron un total de 40 lesiones intestinales donde hubo que realizar sutura intestinal, ya sea asociada o no a resección intestinal; 34 del sexo masculino y 6 del sexo femenino. La edad promedio encontrada fue de 30 años (2/72) (G ráficos 1 and 2).

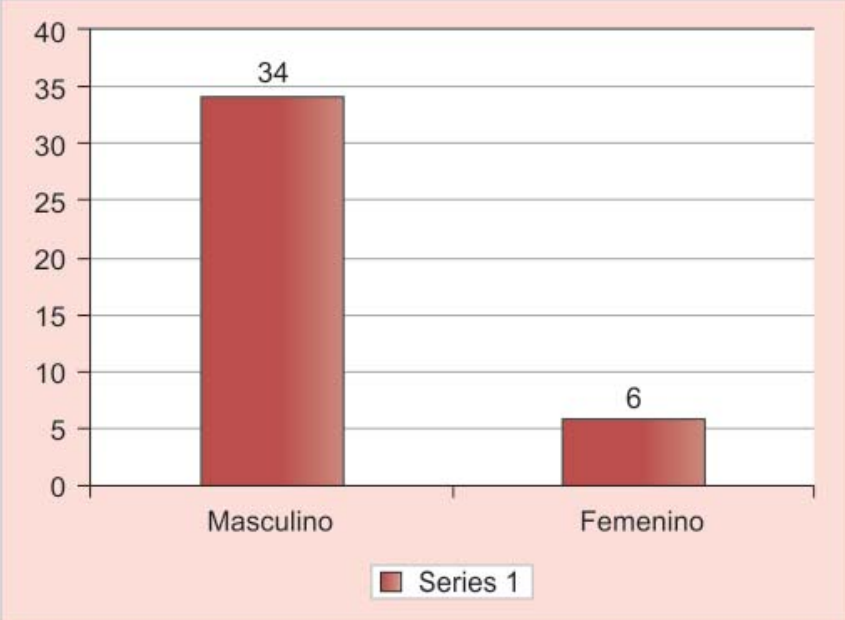

Gráfico 1: Distribución por sexo $(\mathrm{N}=40)$

Según causa: La gran mayoría fue secundaria a accidentes de tránsito $(65 \%)$ causa menos frecuentes fueron caída de bicicletas ( $10 \%)$, caídas (7,5\%); y otros (ver Gráfico 2).

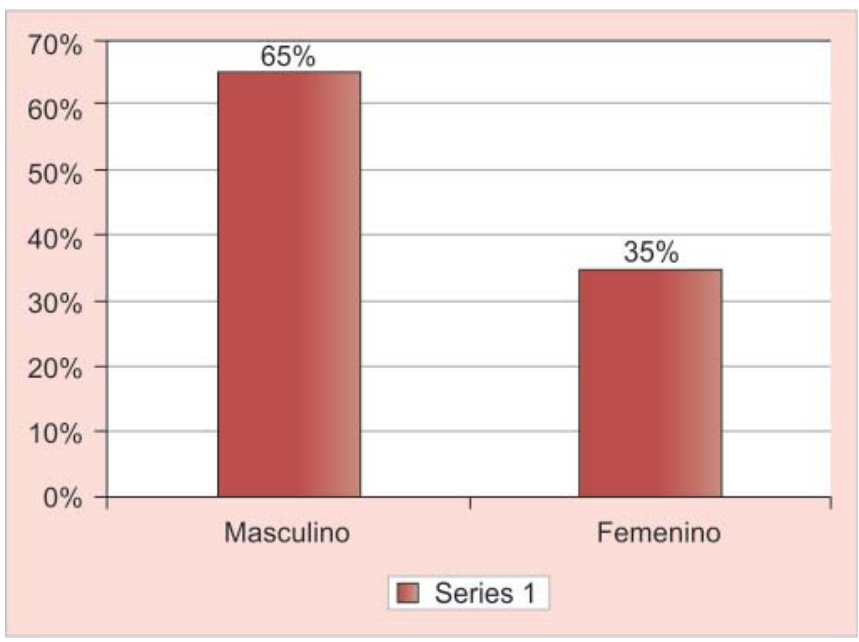

Gráfico 2: Distribución por sexo $(\mathrm{N}=40)$

De los 40 casos citados como lesiones con suturas hubo lesión intestinal abierta por el agente traumático en la mayoría de los pacientes y en menos casos lesión mesentérica, que por la devascularización segmentaria, obligó a resecar el intestino con o sin anastomosis primaria Gráfico 3.

Según tipo de lesión: se encontró mayor porcentaje de lesiones abiertas del intestino delgado $(62,5 \%)$ y de colon (37,5 \%) Gráfico 4.

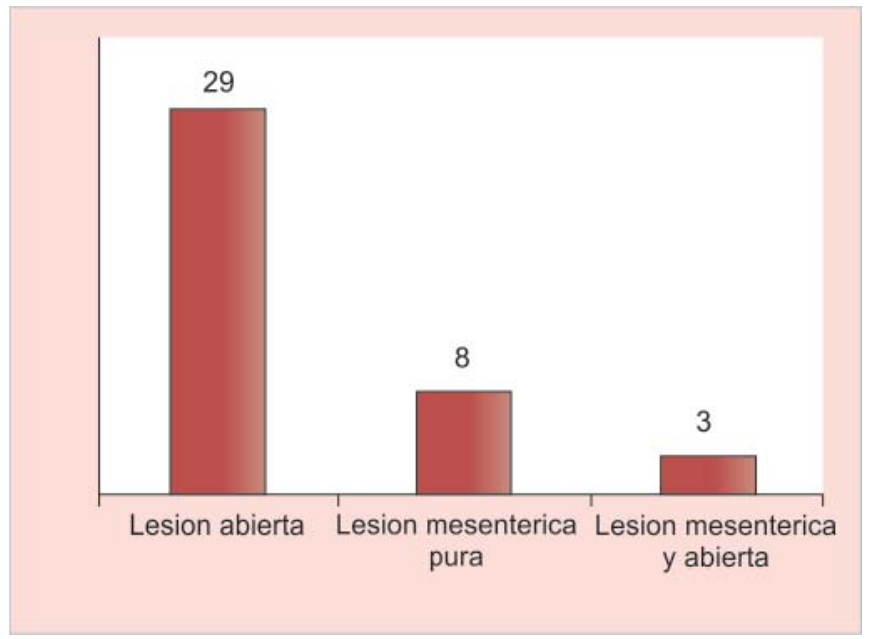

Gráfico 3: Característica de lesión ( $N=40)$

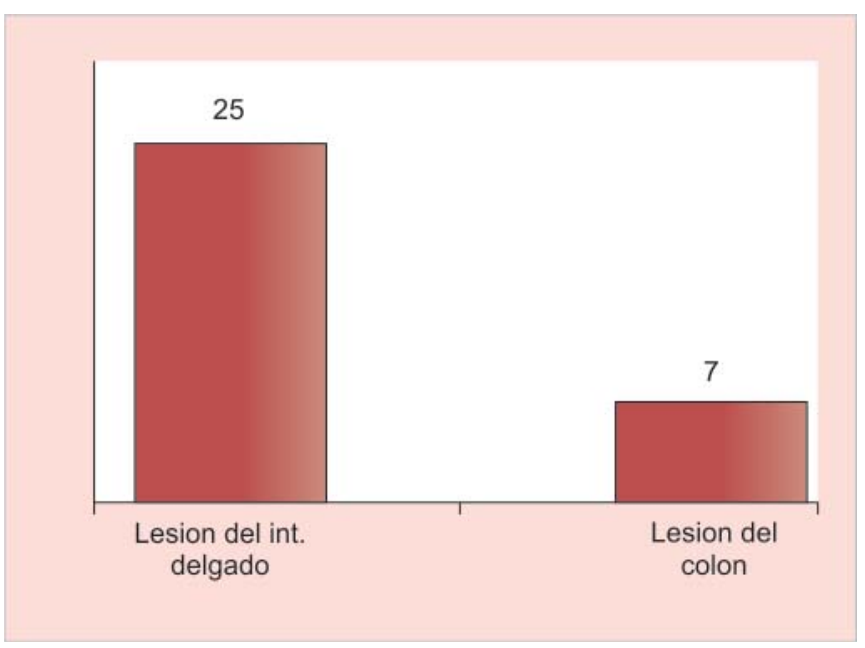

Gráfico 4: Lesiones abiertas $(\mathrm{N}=32)$

Según la mortalidad: Las lesiones abiertas del colon tuvieron un al to porcentaje de mortalidad, mucho mayor en relación a las del intestino delgado ( $G$ ráficos 5 y 7).

Tiempo de evolución desde la cirugía hasta el óbito: La distribución fue como sigue.

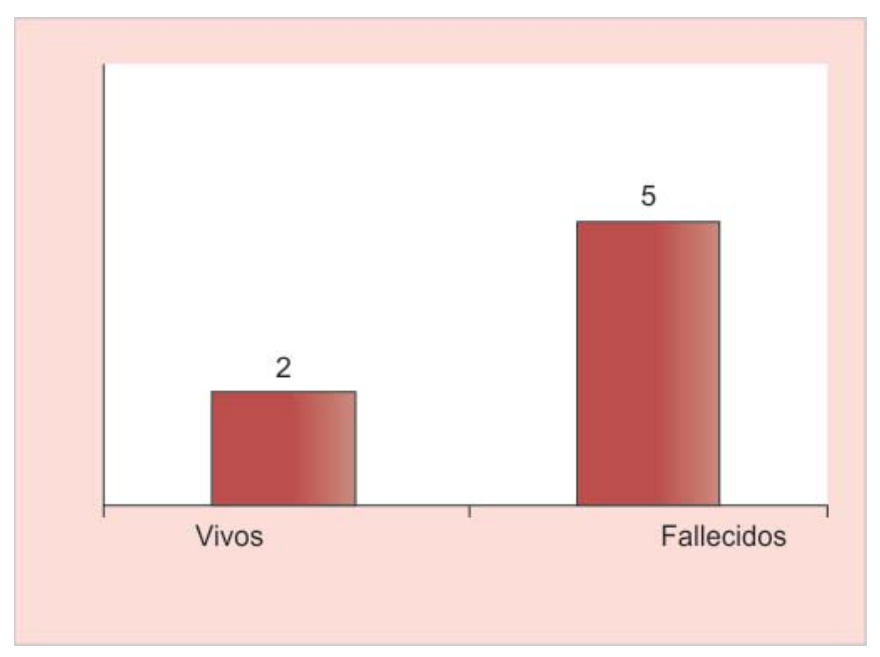

Gráfico 5: Mortalidad en lesión abierta de colon $(\mathrm{N}=7)$ 


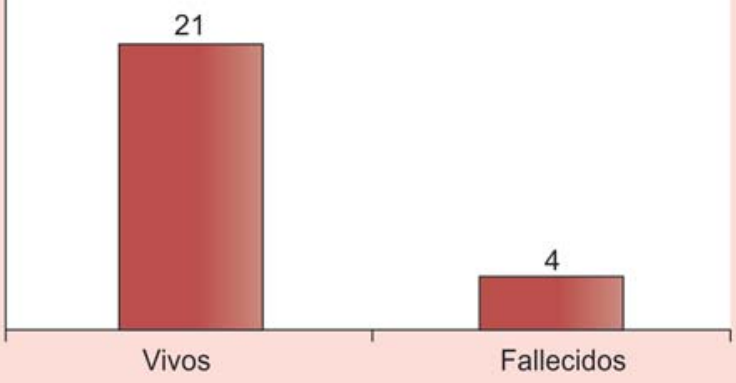

Gráfico 6: Mortalidad en lesiones abiertas de intestino delgado $(\mathrm{N}=25)$

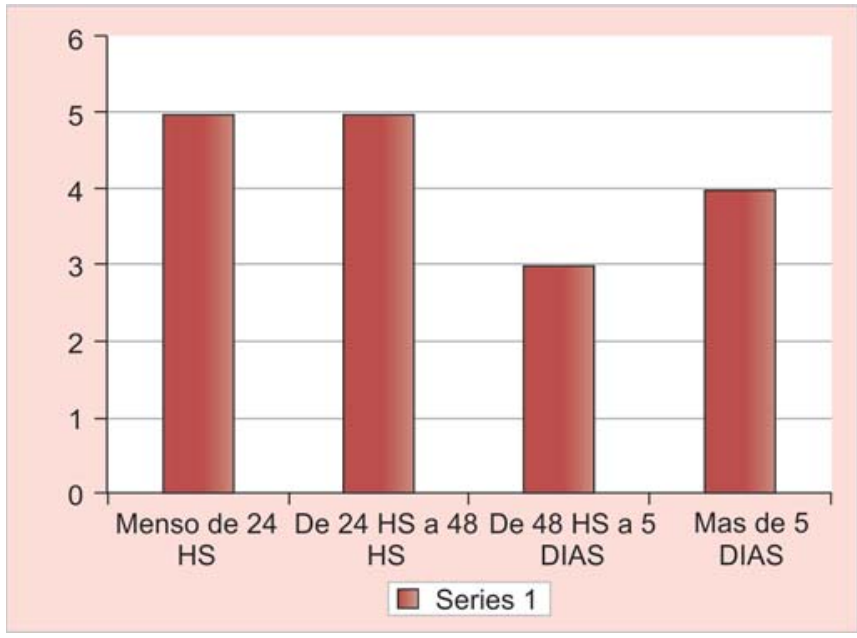

Gráfico 7: Mortalidad según post operatorio $(N=17)$

La relación del tiempo de cirugía al óbito delató que la mayoría se produjo dentro de las 48 hs del postoperatorio (10 de 17), pudiéndose entender por esta característica que las complicaciones antes que ser de fondo séptico se debieron a causa, no relacionada a complicación del manejo intestinal: así encontramos como causa especifica de muerte en los pacientes fallecidos en las primeras 48 hs; shock hemodinámico en 7 , shock séptico 2 y en un paciente se hizo el diagnostico de infarto agudo de miocardio.

En esta serie no se abordaron cl asificación discriminada de lesiones intestinales ni resultados del manejo operatorio y peri-operatorio.

\section{DISCUSION}

Las lesiones intestinales en trauma abdominal contuso en general son referidas como más frecuentemente causadas por accidentes de tránsito, esta presentación ya es sugerida por varios autores y coincide con lo encontrado en este trabajo, además es fuerte la asociación con la ingestión de estimulantes y o bebidas alcohólicas en el contexto de accidentes de vehículos automotores. ${ }^{1,5-7}$

La edad de presentación habitualmente también es referida a personas jóvenes coincidente al hallazgo del trabajo; y el sexo mayormente involucrado el masculino que es recordado como más vulnerable a este tipo de trauma por las actividades que desempeña, así como su conducta de oponer resistencia 0 actuar con violencia en conflictos interpersonales. ${ }^{6}$

Durante la recopilación de datos se encontró además como dato llamativo la presencia de lesión mesentérica pura en 8 pacientes que obligó a la resección intestinal por la isquemia mesentérica que produjo, a este respecto autores como Garret ${ }^{7}$ y Donouhe ${ }^{8}$ han reportado casos de fibrosis de todas las capas en pacientes que debido a injurias mesentéricas sin lesión isquémica distal y que luego se han presentado con diferentes grados de obstrucción intestinal en etapas distales a la fecha de trauma. El diagnostico pre-operatorio de la lesión mesentérica pura es en extremo difícil si no se complementa con estudios invasivos como la laparoscopia o laparotomía. ${ }^{9}$

Existe coincidencia además que las lesiones múltiples son la regla en casos de este tipo de trauma y que la mortalidad por lo general se asocia comúnmente a la inestabilidad hemodinámica y aquí recordamos a B urch que concluye que la mortalidad aumenta de forma importante cuando en el shock se mantiene en forma sostenida en el pre e intra-operatorio. ${ }^{5}$

\section{CONCLUSIÓN}

- Las lesiones intestinales en trauma contuso pueden requerir procedimientos de sutura y/o resección intestinal ya por su apertura a cavidad abdominal libre o por comprometimiento de los vasos mesentéricos.

- En contusiones abdominales es más probable la lesión del intestino delgado que del colon (encontrándose esta relación cercana a 4 por 1 ).

- La mortalidad de la serie está mayormente asociada a lesiones extra-intestinales que debidas directamente a causa del traumatismo intestinal.

\section{REFERENCIAS}

1. K imball I, Rodriguez F. Trauma de estomago e intestino delgado. En: Rodriguez A; Ferrada R (Eds). Trauma. Feriva. Colombia 1997;335-42.

2. Rowlands $B$ J . Intestinal injury due to nonpenetrating abdominal trauma. Injury: B ritish J ournal A ccident Surgery 1984;16:131-33.

3. M aull K I, R eath DB, I mpact of early recognition on outcome in nonpenetranting wounds of the small bowel. Southern M edical J ournal 1984;77(9)1075-77.

4. Sherck JP, Oakes DD, intestinal injuries missed by computed tomography. Journal of Trauma 1990;30:1-7. 
Resecciones y Suturas en el Trauma Intestinal Contuso

5. M achain G. Prevalencia de lesiones colónicas en el traumatismo abdominal. An Fac C M ed (A sunción) 2001;17-46.

6. Palacios F, et al. Experiencia de 5 años en un hospital de tercer nivel en pacientes con trauma abdominal penetrante. Rev. de especialidades médico-quirúrgicas. M éjico 2004:29-33.

7. Garret JW, B raunstein PW. TheSEAT belt syndrome. J Trauma. 2002;2:220-28.

8. Donouhe JH, Federle M P, G riffiths B G, Trunnkey DD. Computed tomography in the diagnosis of blunt intestinal and mesenteric injuries. J Trauma 1997;27: 11-17.

9. Olavarrieta JR. L esiones del mesenterio como consecuencia del trauma abdominal contuso. RFM v.28 n.2 Caracas 2005 Jun: 234-42.
ACERCA DE LOS AUTORES

\section{O Paredes (Correspondencia)}

Emergency Medical Services, A suncion, Paraguay, e-mail: capopuma@hotmail.com

\section{Madelaire}

Emergency M edical Services, A suncion, Paraguay

\section{J Est Domínguez}

Emergency M edical Services, A suncion, Paraguay 